\title{
BMJ Open A multicentre randomised, 1-year comparative effectiveness, parallel- group trial protocol of a physical therapy approach compared to corticosteroid injections
}

Gail D Deyle, ${ }^{1}$ Norman W Gill, ${ }^{1}$ Daniel I Rhon, ${ }^{2}$ Chris S Allen, ${ }^{1}$ Stephen C Allison, ${ }^{1}$ Ben R Hando, ${ }^{3}$ Evan J Petersen, ${ }^{4}$ Douglas I Dusenberry, ${ }^{1}$ Nicholas Bellamy ${ }^{5}$

To cite: Deyle GD, Gill NW, Rhon DI, et al. A multicentre randomised, 1-year comparative effectiveness, parallel-group trial protocol of a physical therapy approach compared to corticosteroid injections. BMJ Open 2016;6: e010528. doi:10.1136/ bmjopen-2015-010528

- Prepublication history and additional material is available. To view please visit the journal (http://dx.doi.org/ 10.1136/bmjopen-2015010528).

GDD, NWG, DIR, SCA and $\mathrm{BRH}$ contributed equally to this work.

Received 23 November 2015 Revised 9 February 2016 Accepted 1 March 2016

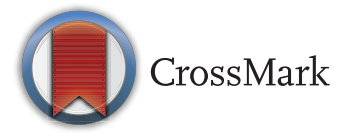

For numbered affiliations see end of article.

Correspondence to Dr Gail D Deyle; gail.d.deyle.civ@mail.mil

\section{ABSTRACT}

Introduction: Corticosteroid injections (CSIs) are commonly used as an initial or a primary intervention for knee osteoarthritis (OA). Consistent evidence indicates CSIs offer symptom relief with conflicting reports regarding long-term efficacy. Physical therapy (PT) offers a non-invasive alternative. There is moderate evidence suggesting short-term and longterm symptom relief and functional improvement with PT interventions. Patients with knee OA are more commonly prescribed CSI than PT prior to total joint replacement. UnitedHealthcare and Military Health System data show substantially more total knee replacement patients receive preoperative CSI than PT. There are no studies comparing CSI to a PT approach in individuals with knee $\mathrm{OA}$. The primary objective of this study is to compare the effectiveness of CSI to PT in individuals with knee $O A$ at 1,2 and 12 months.

Methods and analysis: We plan to recruit 156 participants meeting established knee $\mathrm{OA}$ criteria. Following informed consent, participants will be randomised to receive either CSI or PT. All participants will receive instruction on recommended exercise and weight control strategies plus usual medical care. The CSI intervention consisting of 3 injections and the PT intervention consisting of 8-12 sessions will be spaced over 12 months. Measures of the dependent variables (DVs) will occur at baseline, 4 weeks, 8 weeks, 6 months and 12 months post enrolment. This pragmatic, randomised clinical trial will be a mixedmodel $2 \times 5$ factorial design. The independent variables are treatment (CSI and PT) and time with five levels from baseline to 1 year. The primary DV is the Western Ontario \& McMaster Universities Arthritis Index (WOMAC). We will also compare healthcare utilisation between the 2 groups.

Ethics and Dissemination: The protocol was approved by the Madigan Army Medical Center Institutional Review Board. The authors intend to publish the results in a peer-reviewed source. Trial Registration Number: NCT01427153.

\section{Strengths and limitations of this study}

- This study compares two currently prescribed interventions for knee osteoarthritis (OA). The more commonly prescribed corticosteroid injection (CSI) is more expedient, requiring little patient participation. A CSI may have a limited period of benefit and entails the risk of an invasive procedure. The physical therapy (PT) intervention may offer more durable relief and greater functional improvement. It involves virtually no risk while requiring greater patient participation.

- The PT approach requires tailoring of the examination and treatment to the individual patient with knee $O A$. While this is typical of pragmatic clinical care, it complicates descriptions of the intervention used for this arm of the trial. Careful recording of delivered treatments, precise writing and appropriate use of figures and tables will be required to accurately communicate the intervention details.

- Little is currently known about the utilisation of healthcare resources after either of these two interventions for patients with knee $O A$. This study will help meet recent calls for patientfocused pragmatic management strategies for this complex and debilitating disorder. It may also help initiate additional research comparing pharmacological and non-pharmacological and invasive versus non-invasive approaches.

\section{INTRODUCTION}

Osteoarthritis (OA) of the knee is an increasingly prevalent disorder utilising substantial healthcare resources. ${ }^{1}{ }^{2}$ Management strategies vary greatly according to how patients are routed through the healthcare system and the number and type of providers consulted. Patient presentations are also variable because knee OA can be linked with 
athletic injury, ${ }^{3-5}$ knee trauma, ${ }^{6}$ obesity, ${ }^{1} 78$ occupational or sport overuse, ${ }^{9}{ }^{10}$ female gender, ${ }^{11}$ axial malalignment ${ }^{12}{ }^{13}$ and advanced age. ${ }^{13}$ Knee OA is increasingly viewed as a continuum with early articular cartilage changes found in younger patients predicting progression to degenerative arthritis. ${ }^{13}$ The presence of early radiographic knee $\mathrm{OA}$ predicts progression to more advanced grades of arthritis. ${ }^{11}{ }^{15} 16$ Impairments to movement, strength and flexibility starting early in the degenerative process may accelerate arthritic changes and contribute to the disability associated with knee OA. ${ }^{17} 18$ Rheumatologists, orthopaedic surgeons and family practice physicians commonly treat knee OA with corticosteroid injection (CSI). ${ }^{19}{ }^{20}$ An epidemiological analysis of interventions delivered to patients with knee $\mathrm{OA}$ in the $\mathrm{USA}^{21}$ revealed that of the 11 million patients in the database, 12806 had total knee arthroplasty (TKA) in 2009. Over the 5-year period (2004-2009), $43.5 \%$ of the patients eventually receiving TKA were prescribed preoperative intra-articular steroid injections. Conversely, only $10 \%$ of TKA recipients received physical therapy (PT) intervention of any kind for their knee OA prior to knee replacement. ${ }^{21} \mathrm{~A}$ recent retrospective review of Military Health System (MHS) data similarly shows that only $8 \%$ of participants received PT prior to total joint arthroplasty. ${ }^{22}$ There is substantial evidence for CSI providing short-term pain relief ${ }^{2324}$ and limited evidence for more durable benefit up to 12 months. ${ }^{23} 24$ A recent systematic review and meta-analysis found intra-articular injections, including CSI, to be superior to oral non-steroidal anti-inflammatory drug (NSAID) medications at reducing pain in individuals with knee OA. ${ }^{25}$ The American Academy of Orthopaedic Surgeons, ${ }^{26}$ the American College of Rheumatology ${ }^{27}$ and the 2014 Orthopaedic Research Society International Guidelines recommend the use of CSI as an initial management strategy for treating individuals with knee OA. ${ }^{28}$ Longer term relief may be derived by the pragmatic practice of providing injections in a series, spaced a few months apart. Although CSI is frequently administered prior to PT referrals, a recently published study on patients with knee OA found no additional benefit from CSI compared to placebo injection before a course of supervised exercise at 2,14 or 26 weeks. ${ }^{29}$ While CSIs are generally considered to be standard of care and low risk, there are reports of septic arthritis ${ }^{28} 30$ and other systemic complications linked to intra-articular injections. ${ }^{31}{ }^{32}$ Preoperative, intra-articular CSI links to deep wound infections post-TKA are controversial. $^{28}{ }^{32}$ One group of authors attempted to find factors predicting success with CSI. Their preliminary findings identified factors such as the presence of an effusion and the absence of synovitis at the time of the injection, which may help define patients likely to respond. ${ }^{33}$

PT interventions for knee OA provide symptomatic and functional improvement with low risk. ${ }^{34-39}$ On the basis of previous clinical trials, eight PT sessions consisting of manual PT (MPT) (see Definitions section) with reinforcing clinical and home exercise for patients with knee OA resulted in symptomatic relief and functional improvement up to 1 year and reduced the incidence of total joint replacement, intra-articular injection and medication use. ${ }^{35}$ A single session of MPT improves terminal knee extension, ${ }^{40}$ reduces hyperalgesia and improves Timed Up and Go (TUG) performance in patients with knee OA. ${ }^{41}$ Composite data from two clinical trials revealed that $80 \%$ of patients receiving MPT as a primary intervention for their knee OA sustained greater than the minimal clinically important difference (MCID) level of benefit and $60 \%$ of patients derived greater than $50 \%$ improvement in their baseline Western Ontario \& McMaster Universities Arthritis Index (WOMAC) scores at 4 weeks. ${ }^{35} 3642$ These patients remained improved over baseline at 1 year with no further contact after the initial 4 weeks of treatment.

No studies have compared the intervention strategies of CSI and PT. Therefore, the purpose of this randomised clinical trial will be to directly compare the shortterm and long-term effects of these interventions for patients with knee OA. Our hypothesis is that PT will provide similar symptomatic relief to CSI in the short term with greater functional improvement and more durable symptom relief as evidenced by WOMAC scores at 12 months. A secondary purpose is to compare the knee-related healthcare utilisation in both groups for the 12 months post the final measure of the dependent variables (DVs).

\section{Specific aims}

Aim 1: Compare 1-year self-reported outcomes of pain, function and disability between patients that received CSI and those that received PT.

Aim 2: Compare knee-related healthcare utilisation in both groups during the 1-year follow-up period.

\section{TRIAL DESIGN AND METHODS}

The study was designed using the Standard Protocol Items: Recommendations for Interventional Trials (SPIRIT) guidelines for planning interventional clinical trials, and the results will be reported in accordance with the CONsolidated Standards of Reporting Trials (CONSORT) guidelines for pragmatic trials. ${ }^{43}$ Independent variables are treatment (CSI and PT) and time with five levels from baseline to 1 year. The primary DV is the WOMAC. The secondary DVs are the Global Rating of Change (GROC) and the TUG.

\section{Participants}

We will recruit 156 participants, male and female, aged 38 years or older with a diagnosis of knee OA. These participants will be drawn from patients referred to the PT and orthopaedic services at two teaching medical centres within the US MHS. Training will be given to 
providers in primary care clinics at these centres to inform them of the study and facilitate the flow of patients. Beneficiaries of the MHS include active duty and retired service members and their non-military family members, thereby reflecting the spectrum of patients likely to be seen in a civilian setting. Providers will have a copy of the study screening form and screen interested patients against the inclusion and exclusion criteria (boxes 1 and 2). Once it is determined that an interested patient meets the criteria for inclusion, the site study coordinator will perform the informed consent process. If recruitment targets are not met despite these efforts, flyers with information about the study will be distributed at key locations so that eligible interested participants may inquire. Plans to promote participant retention include informing potential participants that although it is their right to withdraw at any time, simply dropping out without reason weakens the study and reduces the ability to answer this important research question. If participants encounter difficulty attending any appointments or complying with the intervention, they are encouraged to discuss these challenges with the site study coordinator. Potential participants will be additionally guided that if they are uncertain whether they can commit to the study procedures for the entire

\section{Box 1 Inclusion and exclusion criteria}

Inclusion criteria

- Have radiographic evidence of knee $O A$

- Meet Altman's clinical criteria for knee OA (box 2)

- Be 38 years of age or older as per Altman's clinical criteria

- Have English language skills sufficient to complete the WOMAC and GROC outcome instruments

- All participants must be eligible for care in the military health system

Exclusion criteria

- Unable to give informed consent to participate in the study

- Steroid injections or physical therapy treatment for their knee in the past 12 months

- Current or history of gout of the knee

- Active infection in the knee within the past 12 months

- Other physical ailment or condition that is typically more limiting or painful than their knee OA during activities such as sitting, standing, walking or stair climbing

- Current or history of rheumatoid arthritis or similar rheumatic condition

- Cannot speak/read English adequately to understand and provide consent to participate in the study

- Pregnant or intending to become pregnant

- Military service members in a Warrior Transition Unit (WTU) or service equivalent or pending a medical evaluation board/ discharge process. For non-military personnel, anyone that is pending or undergoing any litigation related to their functional status

- Contraindication to receiving a corticosteroid injection (history of allergic or adverse reaction to steroids or steroid injection, history of multiple corticosteroid injections in that area even if not within last year, uncontrolled diabetes mellitus, etc)
Box 2 Altman's clinical criteria for knee OA: One of the three categories below (sensitivity of $89 \%$ and specificity of $88 \%)^{4}$
A. Knee pain for most days of the prior month and
1. Crepitus with active motion (and)
2. Morning stiffness in knee $\leq 30$ min (and)
3. Age $\geq 38$
B. Knee pain for most days of the prior month and
4. Crepitus with active motion (and)
5. Morning stiffness in knee $>30$ min (and)
6. Bony enlargement
C. Knee pain for most days of the prior month and
7. No crepitus (and)
8. Bony enlargement

12-month period, we will gladly provide care for their disorder and not enrol them. When enrolled participants miss scheduled appointments, they will be contacted by the appointment coordinators for rescheduling at a suitable time.

\section{Randomisation}

A random number generator will be used to establish randomisation lists prior to the initiation of the study. Separate randomisation lists will be generated for each participating site. Individual randomised assignments will be concealed according to the following procedure. The group assignment will be recorded on an opaque label that is folded in half such that the label with the participant's group assignment will be on the inside of the fold. The folded opaque label will then be placed inside an opaque sequentially numbered envelope, and the envelope will be sealed. This will prevent the possibility of visualising the treatment group assignment through the sealed envelope. After determining that the inclusion and exclusion criteria are met and the participant provides informed consent, demographic information and the baseline measures of the DVs will be recorded. On completing these processes, an investigator who will not perform assessments of the DVs or other measures will open the next in sequence randomisation envelope indicating the patient's treatment group assignment that will be linked to the patient's unique study identification number.

\section{Blinding}

Investigators blind to the group of assignment and working in areas remote to where the CSI or PT interventions are performed, thereby making it unlikely that they would observe any provider-participant interaction, will perform the measurements and outcome assessments. Participants will be initially instructed and reminded when scheduled for each measurement appointment not to discuss their intervention or group of assignment during the measurement session. Incidents of unmasking will be recorded in the 
participant research folder by measurement session and will be reported as part of the research findings. The statistician will be blinded to the group origin of the analysed data. Participants are accurately informed during the informed consent process that both interventions are standard of care with evidence supporting both. Participants are also informed that it is not known which intervention is better over a short or longer term. Participant outcome expectations for each treatment will be assessed prior to randomisation. Unblinding will occur only in exceptional circumstances when knowledge of treatment group is essential for further management of the patient.

\section{Interventions}

Prior to the randomised assignment, all participants will receive formal 1:1 interactive instruction on the benefits of weight reduction, low-impact, well-tolerated physical activity, and strengthening and flexibility exercises. Participants are then randomised to either the CSI or PT group. The two treatment options are standard-of-care interventions. Both treatments are summarised in table 1 . The flow of participants through both arms of the trial is illustrated in figure 1. If participants in either group request to stop treatment or are demonstrating marked worsening of symptoms, they will be re-evaluated and the treatment may be modified to meet their needs. Patients are not prohibited from continuing with existing medications or from seeking additional care; however, the additional treatments will be documented and reported. Continued care after the end of the study will be offered to all participants.

\section{CSI procedures}

The goal of the CSI is to decrease intra-articular inflammation and pain, thereby improving function and activity tolerance. ${ }^{23} 45$ The CSI will be given by orthopaedic surgeons, orthopaedic physician assistants or rheumatologists consistent with current standards for intra-articular injections. These specialty-trained providers routinely treat patients with chronic musculoskeletal conditions including knee OA. The provider will interview the patient and perform the appropriate clinical examination and health screening to confirm the presence of $\mathrm{OA}$ and the absence of any contraindications to CSI. If the patient meets all inclusion criteria, verbal consent for the procedure will be obtained after educating the patient about the current evidence supporting CSI and possible adverse effects. One or both knees will be injected with $1 \mathrm{cc}$ of $40 \mathrm{mg} / \mathrm{mL}$ of triamcinolone acetonide ${ }^{47}$ and $7 \mathrm{cc}$ of $1 \%$ of lidocaine using sterile technique. Patients will be observed for $15 \mathrm{~min}$ post the procedure with documentation of their physiological and symptom response. As per standard procedure, patients are also guided that they may use ice, heat or anti-inflammatory agent to reduce any post-injection increase in symptoms. They will be advised to avoid strenuous activity or exercise for $72 \mathrm{~h}$ and resume normal activities as tolerated thereafter. Participants in the CSI group receiving the initial injection without any complication will have the option, similar to the option for additional therapy sessions in the PT group, of receiving additional treatment in the form of an additional CSI at the 4-month and 9-month follow-up visits for a maximum total of three injections.

\section{PT procedures}

The PT interventions in this trial will be pragmatic, individualised and patient centered ${ }^{35} 364849$ using MPT interventions with reinforcing exercises. The interventions will be guided by clinical reasoning (see Definitions section $)^{50}{ }^{51}$ to appropriately select, dose and progress them (figure 2). At the first session, the physical therapist will perform a detailed interview and physical examination to determine symptom location and behaviour; impaired tissues, structures and movement; and functional limitations. This information will determine the appropriate focus, scope and vigour of applied PT interventions. The PT interventions including the manual therapy techniques and reinforcing exercises will be directed primarily to the arthritic knee(s) and secondarily to the hip, lumbar spine and foot and ankle regions if they are judged to be contributing to the overall symptoms, limited movement and disability

\section{Table 1 Treatment schedule and dose}

\begin{tabular}{llll}
\hline & Initial treatment & 4-Month treatment & 9-Month treatment \\
\hline $\begin{array}{l}\text { CSI } \\
\text { group }\end{array}$ & $\begin{array}{l}\text { Education on the benefits of weight reduction, regular } \\
\text { low-impact physical activity, and strengthening and flexibility } \\
\text { exercises }\end{array}$ & $\begin{array}{l}\text { Intra-articular steroid } \\
\text { injection as needed }\end{array}$ & $\begin{array}{l}\text { Intra-articular steroid } \\
\text { injection as needed }\end{array}$ \\
& $\begin{array}{l}\text { An intra-articular steroid injection with postinjection activity } \\
\text { guidance }\end{array}$ & Up to 3 PT clinical & Up to 3 PT clinical \\
PT & Education on the benefits of weight reduction, regular & sessions as needed \\
group & $\begin{array}{l}\text { low-impact physical activity, and strengthening and flexibility } \\
\text { exercises }\end{array}$ & sessions as needed & \\
& $\begin{array}{l}\text { Up to } 8 \text { clinical sessions for physical therapy treatments } \\
\text { consisting of manual therapy and reinforcing exercise }\end{array}$ & & \\
\hline CSI, corticosteroid injection; PT, physical therapy. & &
\end{tabular}




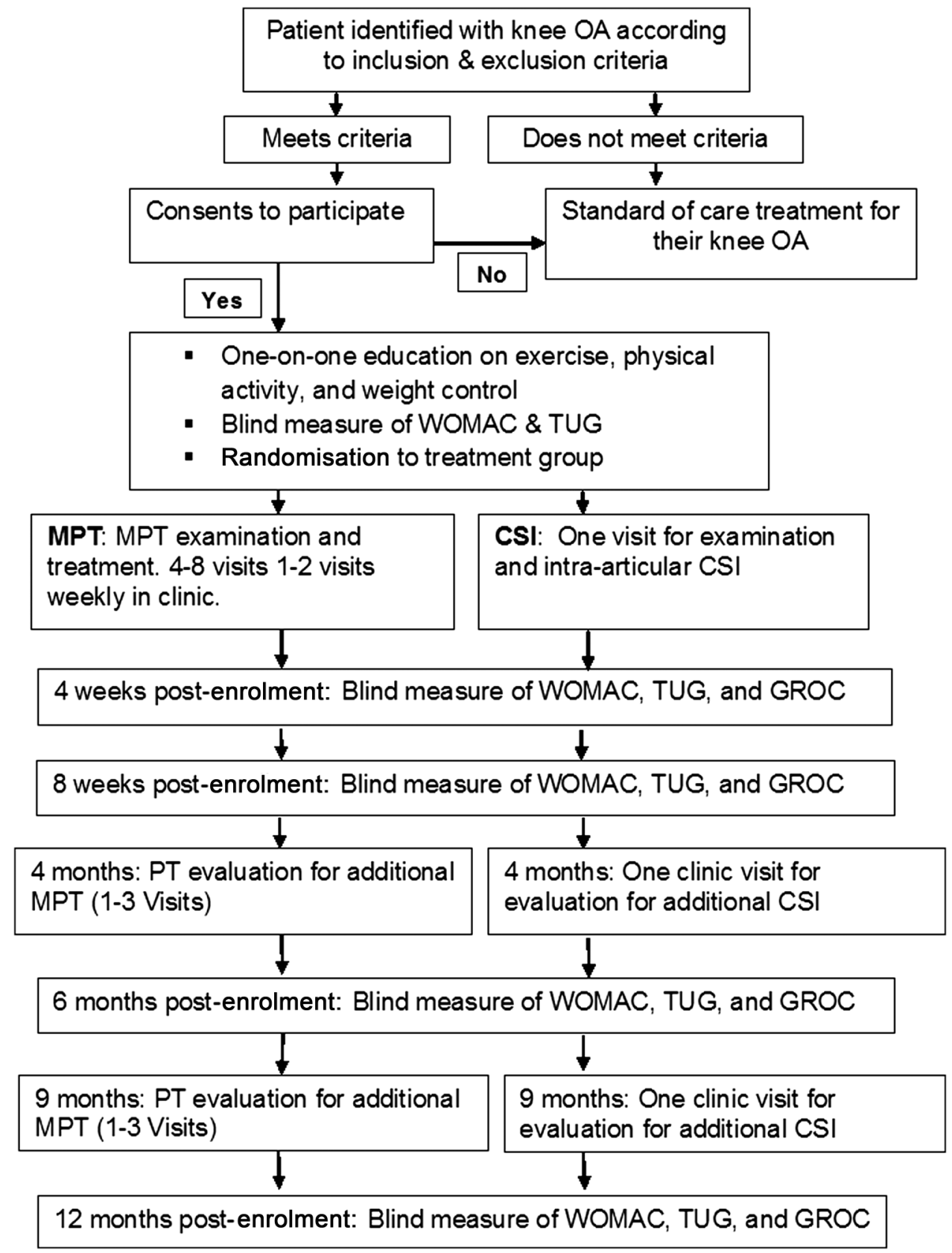

Figure 1 Proposed recruitment and flow of the study. CSI, corticosteroid injection; GROC, Global Rating of Change; MPT, manual physical therapy; OA, osteoarthritis; PT, physical therapy; TUG, Timed Up and Go; WOMAC, Western Ontario \& McMaster Universities Arthritis Index.

the patient attributes to their knee OA. The manual techniques, reinforcing exercises and any other selected exercises will be targeted to impairments in movement, strength, flexibility and motor control identified during the examination. ${ }^{34-36} 48$ The priority, dosing and progression of all treatment will be determined by the treating physical therapist. Accordingly, interventions will not be predetermined or artificially constrained by a protocol that assumes identical treatment is appropriate for all participants despite typically wide variance in clinical presentation. $^{48}$ In this pragmatic patient-centred approach, clinical decision-making ${ }^{50}$ or clinical reasoning $^{51}$ will be employed to tailor PT interventions according to the information gleaned from the interview and physical examination and from the patient's ongoing response to treatment. ${ }^{48}$ The core set of exercises (see online supplementary appendix 1) that reinforce the effects of manual interventions (see online supplementary appendix 2) or any additionally prescribed exercises will be instructed and made part of the home exercise programme at the appropriate points. Participants will have home exercise folders with pictures and instructions for each exercise, compliance logs to document exercise performance and videos of the core 
Patient consented and randomised to MPT group

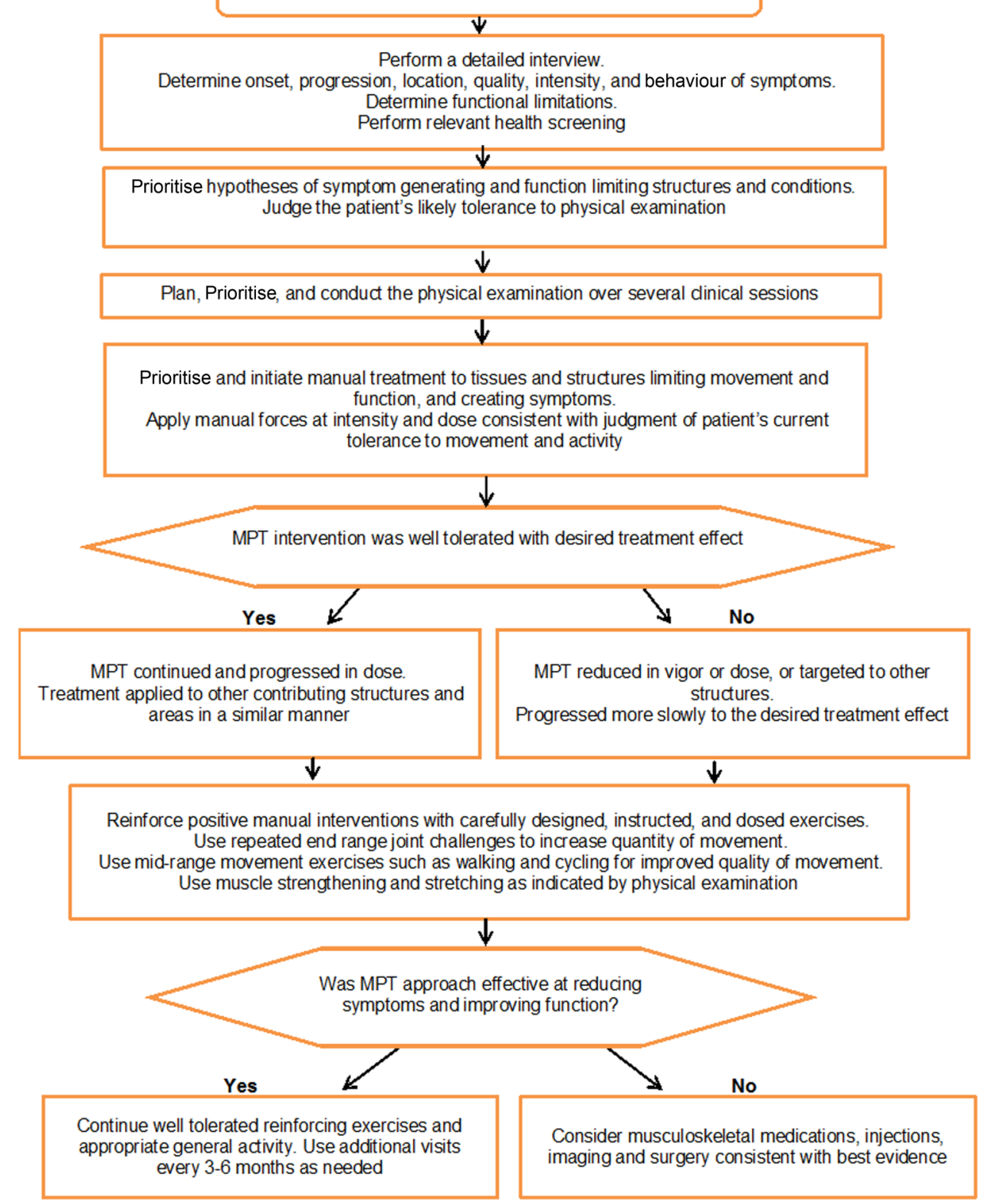

Figure 2 PT approach algorithm. MPT, manual physical therapy; PT, physical therapy.

set of exercises to enhance compliance and accurate performance of the exercises. PT patients in this setting routinely receive similar home exercise folders as standard of care. Participants are assessed before, during and after treatment sessions to determine the effects of manual techniques and exercises on symptoms and function. Each participant will initially receive a maximum of eight PT sessions over a 4-6-week period. Frequency and duration of treatment is at the discretion of the treating PT. Additional 2-3 PT sessions may be used at the 4-month and 9-month rechecks. Each treatment session will last approximately $60 \mathrm{~min}$. A physical therapist will perform all treatments including exercise instruction and supervision. Treatments will be progressed according to the patient's response to the prior session(s). At the beginning of each session, patients who are cycling tolerant will warm up on an appropriately fitted stationary bicycle for $10-12 \mathrm{~min}$ prior to receiving manual examination and treatment. Bicycle sessions will include a progressive number of strengthening or higher intensity power intervals lasting $20 \mathrm{~s}$ followed by $40 \mathrm{~s}$ of recovery spinning. The primary exercises used in this study (see online supplementary appendix 1) address typical strength and movement impairments commonly found in patients with knee OA and reinforce the effects of the clinically applied MPT $^{36}{ }^{48}$ One or two exercises will be added at each session consistent with the priority of identified impairments and the corresponding manual treatment techniques used. This progressive layering approach is useful 
to determine the effects of new techniques and exercises. Each manual technique or exercise will be progressed, modified or eliminated as needed to keep the programme well tolerated ${ }^{48}$ by the patient. Additional exercises targeted to specific impairments such as hip muscle weakness or spine hypomobility may be used depending on examination findings. This approach is consistent with previous MPT trials on participants with knee $\mathrm{OA}^{34-36}$ and chronic conditions in other body regions. ${ }^{52-56}$ A typical progression of manual techniques and reinforcing exercises is illustrated in figure 3. Each patient is evaluated at 4 and 9 months post initiating treatment to determine if there are questions on the home exercises or if additional manual therapy is indicated. The physical therapists providing treatment are board certified in orthopaedic PT and fellowship trained in orthopaedic manual PT. They routinely treat patients with chronic musculoskeletal conditions including knee OA.

\section{Outcome measures}

The primary DV is the WOMAC and the secondary DVs are the GROC and the TUG.

The DVs and other examination data such as height, weight, knee active range of motion, presence of joint line tenderness, ligamentous stability tests and symptoms with patellofemoral passive movement are recorded onto forms within the participant data folder.

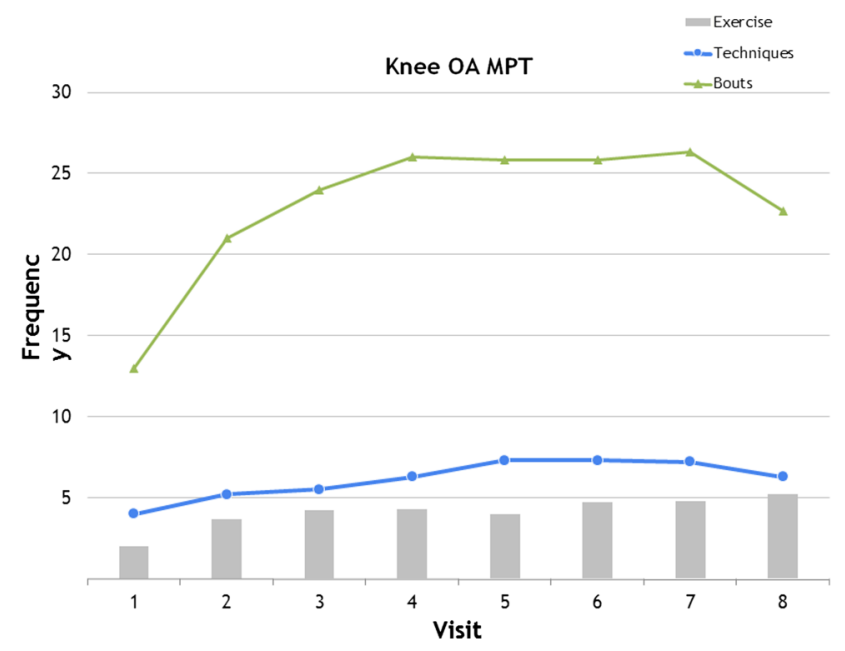

Figure 3 Manual therapy techniques and exercise dose progression. This figure represents a typical MPT and exercise dose progression by visit. Bouts of manual therapy are reported as a single bout of joint or soft tissue mobilisation for $30 \mathrm{~s}$. Techniques are the number of different joint or soft tissue mobilisation techniques used during the visit. Manual therapy dosage and exercise prescription typically increase throughout the course of care as the patient demonstrates tolerance. At the last visits, the dosage of manual therapy typically decreases as the focus shifts towards reinforcing the importance of the patient's long-term performance of the home exercise programme. MPT, manual physical therapy; OA, osteoarthritis.
Participant folders are labelled with participant numbers corresponding to the number from the randomisation lists. Folders are filed sequentially by number and maintained in a locked filing cabinet in a locked room.

\section{The WOMAC}

The WOMAC is a self-report questionnaire requiring patients to rate their pain, stiffness and functional limitation associated with their condition. This instrument will provide important information about the self-reported pain and disability level of the patients in this study. The WOMAC is a recommended primary outcome measure in therapy trials of arthritic conditions ${ }^{57}$ and is considered one of the most appropriate scales for trials evaluating knee OA. ${ }^{58}$ It is a reliable, valid and responsive instrument widely used in clinical trials evaluating therapy for hip and knee OA. ${ }^{59}$

\section{GROC questionnaire}

The GROC questionnaire is an instrument that measures overall changes in the quality of life of the participant. ${ }^{60}$ The GROC is a common, feasible and useful method for assessing outcome ${ }^{61}$ and is a valid measurement of change in patient status in other pain populations. ${ }^{62}$ A change in score of three rating points $(+3)$ is clinically significant in the patient's perception of quality of life. ${ }^{61}$ The GROC has 15 possible choices, with 0 being equal to no change, -1 to -7 indicating a negative change and +1 to +7 indicating a positive change.

\section{TUG test}

The TUG test is a functional performance measure specifically studied in persons with OA of the hip and knee, which directly evaluates an individual's ability to transfer, ambulate and maintain balance during transitions. ${ }^{63-65}$ Individuals are timed on how quickly they can stand, walk $3 \mathrm{~m}$, turn around return to the chair and sit down. The TUG has good inter-rater and intrarater reliability and validity for functional testing in older adults. ${ }^{66-68} \mathrm{It}$ also correlates well with the Berg Balance Scale ( $r=$ $-0.81)$, gait speed $(\mathrm{r}=-0.61)$ and the Barthel Index of Activities of Daily Living $(\mathrm{r}=-0.78) .{ }^{66}$ According to Podsiadlo $e t a l,{ }^{66}$ the TUG is a reliable and valid test for quantifying functional mobility, and it is a good predictor of a patient's ability to go outside safely. It has content validity, evaluating a series of manoeuvers used in daily life, and concurrent validity in that it correlates well with more extensive measures of balance, gait speed and functional abilities. The TUG was used as a functional outcome in a previous MPT trial for knee $\mathrm{OA}^{34}$ and is a currently recommended knee OA functional outcome measure. ${ }^{65}$ The test is easy to administer and can be completed in 2-3 min.

We will also monitor for and report any adverse effects associated with either treatment. In this study, an adverse event will be defined as a persistent worsening of symptoms requiring additional treatment outside of the study. 


\section{Justification of sample size}

A total of 69 participants per group, or a total of 138 participants, are needed for $80 \%$ power to detect a significant group $\times$ time interaction effect using an $\alpha$ level of 0.05 and assuming a $12 \%$ difference ${ }^{69}$ between groups in mean post-treatment total WOMAC scorescorresponding to a partial $\eta^{2}$ of 0.006 and an effect size $f$ of 0.078 . This assumes two groups measured over five points in time, a common SD of 46.8 points, a between-repeated-measures correlation of $r=0.681$, and a non-sphericity correction factor of (Greenhouse-Geisser) epsilon $=0.890$ - consistent with data collected in previous trials. ${ }^{35} 36$ Assuming an $11 \%$ dropout rate, ${ }^{8}$ we will need to enrol 156 (approximately 80 from each of the 2 participating centres) participants in order to have 138 participants completing the study. A sensitivity analysis was performed to compute magnitudes of statistical power reduction given varying scenarios with dropout rates greater than the assumed rate of $11 \%$. If the dropout rate should be $15 \%$ (132 participants retained), with all other power determinants remaining unchanged, statistical power would be reduced to $78 \%$; with a $20 \%$ dropout rate (125 participants retained), statistical power would be $75 \%$; with a $25 \%$ dropout rate (117 participants retained), statistical power would be $72 \%$. Sample size estimation was performed with $G^{*}$ Power software, V.3.1.2. ${ }^{70}$ Recruitment will not go over a total of 156 participants without prior Institutional Review Board (IRB) approval, but a site may have more than their proportionate share depending on clinical flow and available participant pool. In this case, the other site will under-recruit.

\section{Data entry}

Data entered into the research folder will be periodically transferred into the master data spreadsheet by a research assistant. Accuracy of data recording is facilitated by secondary review of data by coauthors. Intervention groups are coded as 1 or 2 to prevent unmasking of datasets during analysis as the statistician will remain blind to the group of assignment. Data from individual participants are recorded by research numbers. The spreadsheet containing the research data is stored on a portable drive and locked in the same room and cabinet as the research folders.

\section{Data analysis}

Data analysis will be performed with IBM SPSS Statistics software, V.22. The statistician performing the analyses will be blinded to the intervention associated with the group of assignment. Descriptive statistics will be computed to characterise and compare the two treatment groups for assessment of baseline heterogeneity. Distributions of measured variables will be examined visually with frequency histograms and formally assessed with Kolmogorov-Smirnov and Shapiro-Wilk statistics to test the normality assumption. Levene's test will be used to assess for violations of the homogeneity of variance assumption. All statistical tests will all be performed at an $\alpha$ level of 0.05 .

The primary analysis of relative effectiveness between the two treatments will be tested using a linear mixed-effects model which is flexible in accommodating data assumed to be missing at random. There will be two levels of treatment and five levels of time if randomisation produces reasonably equivalent groups as measured by important prognostic factors (KellgrenLawrence scores, duration of symptoms, baseline WOMAC scores, etc). Otherwise, variables for which non-equivalence is detected at baseline will be entered as covariates into the linear mixed-effects model. A similar approach will be used to analyse the GROC data. An $\alpha$ level of 0.05 will be used to establish significance. The primary analysis will include data from patients according to the group they were assigned. If one treatment is shown to be superior to the other, supplemental analyses will be performed by dichotomising groups based on MCIDs of $12 \%$ for WOMAC ${ }^{69}$ and +3 points for GROC scores. ${ }^{61}$ This will allow computation of absolute risk reduction, relative risk reduction and number needed to treat (with associated 95\% CIs) using failure to obtain clinically meaningful benefit as the event of interest. Every effort will be made to recruit 156 participants. No interim analysis or alternative analysis is planned. If factors beyond the control of the investigators, such as military reassignment of members of the research team, or other emerging research studies at the same centres on the same population prolong enrolment beyond a reasonable period, enrolment may be terminated and the specified blind analysis performed at that point. Owing to the low risk associated with the interventions, a data monitoring committee will not be used.

\section{Trial organisation and monitoring}

The investigative team consists of the authors listed in this protocol, in addition to physical therapists who will measure the DVs and administrative research staff at each site who will assist with appointment scheduling and data entry. The principal investigator will manage data flow and perform audits of the procedures, enrolment and treatment throughout the entire process of the study. The associate investigators will monitor data collection processes and data integrity with periodic evaluation performed continually during the course of the data collection phase.

\section{DISCUSSION}

This randomised clinical trial will be the first study that directly compares the short-term and long-term effects of CSI and PT for patients with knee OA. This question is important because analysis of civilian and military healthcare databases indicates frequent deviation from clinical practice guidelines and patients with knee OA routinely receive CSI as either an initial or a primary 
intervention. Smaller percentages of patients with knee OA receive PT compared to CSI prior to total joint replacement. ${ }^{21} 22$ Injections are often easier, less expensive and more convenient for patients. However, the convenience and short-term savings may be offset by the associated risks and downstream healthcare utilisation associated with the development of chronically impaired movement, and progressing arthritis leading to increasing symptoms, medication use, imaging and invasive procedures. All described PT treatment procedures including MPT are part of entry-level physical therapist education and training. The results of this study may aid in establishing best clinical practice guidelines for this patient population. A challenge with reporting the methods of this pragmatic study will be to communicate the details of the PT interventions including the MPT in a succinct and clear manner that facilitates understanding for further study by other researchers and integration into clinical practice. In particular, clinical reasoning which individualises the type, progression and dose of the PT intervention is not easily described in limited space. Therefore, accurate tracking and reporting of all interventions as applied in this study through the use of graphs and figures derived from data entered onto the treatment recording form (see online supplementary appendix 3) will provide valuable insight into typical treatment progression. The PT treatment algorithm (figure 2) should be helpful to communicate the decision points in pragmatic PT treatment.

\section{ETHICAL CONSIDERATIONS AND DISSEMINATION}

An ethics review has been provided by the Institutional Review Board at Madigan Army Medical Center and monitored by the US Army Medical Department Clinical Investigation Regulatory Office (CIRO) to ensure compliance with federal regulations for protection of human medical research participants. Any significant modifications to the protocol which may impact the conduct of the study, treatment parameters, study objectives or study procedures will require a formal amendment to the protocol and approval by the Institutional Review Board. This clinical trial was registered with ClinicalTrials.gov with a registration number NCT01427153. All interventions provided in this study are considered standard of care and could be given to patients as part of their treatment plan even if they were not a part of this study. Unfortunately, knee OA patients in some practice settings may not receive PT that is similarly comprehensive.

\section{Publication policy}

The results of the trial will be published in a relevant scientific journal regardless of the outcome. We will report the results following the CONSORT statement with the recommended extension for pragmatic trials. ${ }^{43}$

Projected timetable for trial:

May 2012-Protocol approved by the Western Regional Medical Command Institutional Review Board.

Oct 2012-Participant enrolment begins
Jul 2016-Participant enrolment completes

Oct 2013-First participant completes 1-year follow-up Jul 2017-Last participant completes 1-year follow-up

Dec 2017-Data entry and analysis complete

May 2018-Publication with study results submitted for publication

\section{Definitions}

Clinical reasoning: Clinical reasoning is an ongoing decision-making process used in the evaluation and management of a patient throughout the episode of care. ${ }^{5051}$

MPT: 'Orthopaedic Manual Therapy is a specialised area of Physiotherapy/Physical Therapy for the management of neuromusculoskeletal conditions, based on clinical reasoning, using highly specific treatment approaches including manual techniques and therapeutic exercises'. ${ }^{71}$

\section{Author affiliations}

${ }^{1}$ Brooke Army Medical Center, San Antonio, Texas, USA

${ }^{2}$ Madigan Army Medical Center, Tacoma, Washington, USA

${ }^{3}$ National Defense University, Washington, DC, USA

${ }^{4}$ University of the Incarnate Word, San Antonio, Texas, USA

${ }^{5}$ University of Queensland, Brisbane, Queensland, Australia

Twitter Follow Ben Hando at @benhando

Contributors All the authors collectively conceived the idea for the project. GDD is the site principal investigator (PI) at Brooke Army Medical Center and DIR is the site PI at Madigan Army Medical Center. All authors contributed to the scientific derivation of the protocol, as well as reviewing and submitting the protocol for publication. SCA provided advice with statistical and methods design. GDD, CSA, EJP and DIR will provide all the interventions for the physical therapy (PT) group. NB is the developer of the Western Ontario \& McMaster Universities Arthritis Index (WOMAC) and consultant for the research design and question. Authors NWG and BRH will coordinate appointments for treatment and blinded follow-up testing over the course of the study.

Funding This study is funded in part by the Orthopaedic Physical Therapy Products Grant through the American Academy of Orthopaedic Manual Physical Therapists.

Disclaimer The views expressed are those of the authors and do not reflect the official policy of the Department of the Army, the Department of Defense or the US Government.

\section{Competing interests None declared.}

Ethics approval Western Regional Medical Command Institutional Review Board.

Provenance and peer review Not commissioned; externally peer reviewed.

Data sharing statement Data from the study are available by email request to the lead author for the purpose of systematic review and meta-analysis.

Open Access This is an Open Access article distributed in accordance with the Creative Commons Attribution Non Commercial (CC BY-NC 4.0) license, which permits others to distribute, remix, adapt, build upon this work noncommercially, and license their derivative works on different terms, provided the original work is properly cited and the use is non-commercial. See: http:// creativecommons.org/licenses/by-nc/4.0/

\section{REFERENCES}

1. Murphy LB, Moss S, Do BT, et al. Annual incidence of knee symptoms and four knee osteoarthritis outcomes in the Johnston County Osteoarthritis Project. Arthritis Care Res (Hoboken) 2016;68:55-65.

2. Losina E, Thornhill TS, Rome BN, et al. The dramatic increase in total knee replacement utilization rates in the United States cannot 
be fully explained by growth in population size and the obesity epidemic. J Bone Joint Surg Am 2012;94:201-7.

3. Fitzgerald GK, Piva SR, Irrgang JJ. Reports of joint instability in knee osteoarthritis: its prevalence and relationship to physical function. Arthritis Rheum 2004;51:941-6.

4. Struewer J, Frangen TM, Ishaque B, et al. Knee function and prevalence of osteoarthritis after isolated anterior cruciate ligament reconstruction using bone-patellar tendon-bone graft: long-term follow-up. Int Orthop 2012;36:171-7.

5. Blalock D, Miller A, Tilley M, et al. Joint instability and osteoarthritis. Clin Med Insights Arthritis Musculoskelet Disord 2015;8:15-23.

6. Emery CA, Roos EM, Verhagen E, et al. OARSI Clinical Trials Recommendations: design and conduct of clinical trials for primary prevention of osteoarthritis by joint injury prevention in sport and recreation. Osteoarthr Cartil 2015;23:815-25.

7. Lohmander LS, Gerhardsson de Verdier M, Rollof J, et al. Incidence of severe knee and hip osteoarthritis in relation to different measures of body mass: a population-based prospective cohort study. Ann Rheum Dis 2009;68:490-6.

8. Losina E, Weinstein AM, Reichmann WM, et al. Lifetime risk and age at diagnosis of symptomatic knee osteoarthritis in the US. Arthritis Care Res (Hoboken) 2013;65:703-11.

9. Sandmark H, Hogstedt C, Vingard E. Primary osteoarthrosis of the knee in men and women as a result of lifelong physical load from work. Scand J Work Environ Health 2000;26:20-5.

10. Jarvholm B, From C, Lewold $\mathrm{S}$, et al. Incidence of surgically treated osteoarthritis in the hip and knee in Male construction workers. Occup Environ Med 2008;65:275-8.

11. Kerkhof HJ, Bierma-Zeinstra SM, Arden NK, et al. Prediction model for knee osteoarthritis incidence, including clinical, genetic and biochemical risk factors. Ann Rheum Dis 2014;73:2116-21.

12. Cerejo R, Dunlop DD, Cahue $\mathrm{S}$, et al. The influence of alignment on risk of knee osteoarthritis progression according to baseline stage of disease. Arthritis Rheum 2002;46:2632-6.

13. Madry H, Luyten FP, Facchini A. Biological aspects of early osteoarthritis. Knee Surg Sports Traumatol Arthrosc 2012;20:407-22.

14. Ryd L, Brittberg M, Eriksson $\mathrm{K}$, et al. Pre-osteoarthritis: definition and diagnosis of an elusive clinical entity. Cartilage 2015;6:156-65.

15. Riddle DL, Makowski M, Kong X. Knee osteoarthritis worsening across the disease spectrum and future knee pain, symptoms and functioning: a multisite prospective cohort study. Arthritis Care Res (Hoboken) 2015;67:1722-9.

16. Wolfe F, Lane NE. The longterm outcome of osteoarthritis: rates and predictors of joint space narrowing in symptomatic patients with knee osteoarthritis. J Rheumatol 2002;29:139-46.

17. Hodges PW, van den Hoorn W, Wrigley TV, et al. Increased duration of co-contraction of medial knee muscles is associated with greater progression of knee osteoarthritis. Man Ther 2016;21:151-8.

18. Zeni JA, Rudolph K, Higginson JS. Alterations in quadriceps and hamstrings coordination in persons with medial compartment knee osteoarthritis. J Electromyogr Kinesiol 2010;20:148-54.

19. Hochberg MC, Perlmutter DL, Hudson Jl, et al. Preferences in the management of osteoarthritis of the hip and knee: results of a survey of community-based rheumatologists in the United States. Arthritis Care Res 1996:9:170-6.

20. Horne G, Devane P, Davidson A, et al. The influence of steroid injections on the incidence of infection following total knee arthroplasty. N Z Med J 2008;121:U2896.

21. Dhawan A, Mather RC III, Karas V, et al. An epidemiologic analysis of clinical practice guidelines for non-arthroplasty treatment of osteoarthritis of the knee. Arthroscopy 2014;30:65-71.

22. Rhon D. Army Medical Command, 1-Year Knee-Related Utilization of Patients with Knee Osteoarthritis within the Military Health System from 2008-2013. Available from the Military Health System Data Repository (MDR). http://www.health.mil/Military-Health-Topics/ Technology/Clinical-Support/Military-Health-System-Data-Repository, 2015.

23. Bellamy N, Campbell J, Robinson V, et al. Intraarticular corticosteroid for treatment of osteoarthritis of the knee. Cochrane Database Syst Rev 2006;(2):CD005328.

24. Cheng OT, Souzdalnitski D, Vrooman B, et al. Evidence-based knee injections for the management of arthritis. Pain Med 2012;13:740-53.

25. Bannuru RR, Schmid CH, Kent DM, et al. Comparative effectiveness of pharmacologic interventions for knee osteoarthritis: a systematic review and network meta-analysis. Ann Intern Med 2015;162:46-54.

26. Surgeons $\mathrm{AAOO}$. Appropriate use criteria for non-arthroplasty treatment of osteoarthritis of the knee. 6 December 2013.

27. Hochberg MC, Altman RD, April KT, et al. American College of Rheumatology 2012 recommendations for the use of nonpharmacologic and pharmacologic therapies in osteoarthritis of the hand, hip, and knee. Arthritis Care Res (Hoboken) 2012;64:465-74.

28. Marsland D, Mumith A, Barlow IW. Systematic review: the safety of intra-articular corticosteroid injection prior to total knee arthroplasty. Knee 2014;21:6-11.

29. Henriksen M, Christensen R, Klokker L, et al. Evaluation of the benefit of corticosteroid injection before exercise therapy in patients with osteoarthritis of the knee: a randomized clinical trial. JAMA Intern Med 2015;175:923-30.

30. Shemesh S, Heller S, Salai M, et al. Septic arthritis of the knee following intraarticular injections in elderly patients: report of six patients. Isr Med Assoc J 2011;13:757-60.

31. Ungprasert P, Permpalung N, Summachiwakij S, et al. A case of recurrent acute pancreatitis due to intra-articular corticosteroid injection. JOP 2014;15:208-9.

32. Berthelot JM, Le Goff $B$, Maugars $Y$. Side effects of corticosteroid injections: what's new? Joint Bone Spine 2013;80:363-7.

33. Maricar N, Callaghan MJ, Felson DT, et al. Predictors of response to intra-articular steroid injections in knee osteoarthritis--a systematic review. Rheumatology (Oxford) 2013;52:1022-32.

34. Abbott JH, Robertson MC, Chapple C, et al. Manual therapy, exercise therapy, or both, in addition to usual care, for osteoarthritis of the hip or knee: a randomized controlled trial. 1: clinical effectiveness. Osteoarthritis Cartilage 2013;21:525-34.

35. Deyle GD, Allison SC, Matekel RL, et al. Physical therapy treatment effectiveness for osteoarthritis of the knee: a randomized comparison of supervised clinical exercise and manual therapy procedures versus a home exercise program. Phys Ther 2005;85:1301-17.

36. Deyle GD, Henderson NE, Matekel RL, et al. Effectiveness of manual physical therapy and exercise in osteoarthritis of the knee. A randomized, controlled trial. Ann Intern Med 2000;132:173-81.

37. Katz JN, Losina E. Surgery versus physical therapy for meniscal tear and osteoarthritis. N Engl J Med 2013;369:677-8.

38. Jansen MJ, Viechtbauer W, Lenssen AF, et al. Strength training alone, exercise therapy alone, and exercise therapy with passive manual mobilisation each reduce pain and disability in people with knee osteoarthritis: a systematic review. J Physiother 2011;57:11-20.

39. Page CJ, Hinman RS, Bennell KL. Physiotherapy management of knee osteoarthritis. Int $J$ Rheum Dis 2011;14:145-51.

40. Taylor AL, Wilken JM, Deyle GD, et al. Knee extension and stiffness in osteoarthritic and normal knees: a videofluoroscopic analysis of the effect of a single session of manual therapy. $J$ Orthop Sports Phys Ther 2014;44:273-82.

41. Moss $P$, Sluka $K$, Wright $A$. The initial effects of knee joint mobilization on osteoarthritic hyperalgesia. Man Ther 2007:12:109-18.

42. Deyle GD, Gill NW, Allison SC, et al. Manual physical therapy and exercise improves knee $\mathrm{OA}$ : Who are the minority unlikely to benefit? A preliminary clinical prediction rule. J Fam Pract 2012;61: E1-8.

43. Zwarenstein M, Treweek S, Gagnier JJ, et al. Improving the reporting of pragmatic trials: an extension of the CONSORT statement. BMJ 2008;337:a2390

44. Altman RD. Criteria for the classification of osteoarthritis of the knee and hip. Scand J Rheumatol Suppl 1987;65:31-9.

45. Leopold SS, Redd BB, Warme WJ, et al. Corticosteroid compared with hyaluronic acid injections for the treatment of osteoarthritis of the knee. A prospective, randomized trial. J Bone Joint Surg Am 2003;85-A:1197-203.

46. Arroll B, Goodyear-Smith F. Corticosteroid injections for osteoarthritis of the knee: meta-analysis. BMJ 2004;328:869.

47. Hepper CT, Halvorson JJ, Duncan ST, et al. The efficacy and duration of intra-articular corticosteroid injection for knee osteoarthritis: a systematic review of level I studies. J Am Acad Orthop Surg 2009;17:638-46.

48. Deyle GD, Gill NW. Well-tolerated strategies for managing knee osteoarthritis: a manual physical therapist approach to activity, exercise, and advice. Phys Sportsmed 2012;40:12-25.

49. Bennell KL, Dobson F, Hinman RS. Exercise in osteoarthritis: moving from prescription to adherence. Best Pract Res Clin Rheumatol 2014;28:93-117.

50. Wainwright SF, Shepard KF, Harman LB, et al. Factors that influence the clinical decision making of novice and experienced physical therapists. Phys Ther 2011;91:87-101.

51. Edwards I, Jones M, Carr J, et al. Clinical reasoning strategies in physical therapy. Phys Ther 2004;84:312-30; discussion 31-5. 
52. Walker MJ, Boyles RE, Young BA, et al. The effectiveness of manual physical therapy and exercise for mechanical neck pain: a randomized clinical trial. Spine 2008;33:2371-8.

53. Whitman JM, Flynn TW, Childs JD, et al. A comparison between two physical therapy treatment programs for patients with lumbar spinal stenosis: a randomized clinical trial. Spine 2006;31: 2541-9.

54. Cleland JA, Abbott JH, Kidd MO, et al. Manual physical therapy and exercise versus electrophysical agents and exercise in the management of plantar heel pain: a multicenter randomized clinical trial. J Orthop Sports Phys Ther 2009;39:573-85.

55. Cleland JA, Mintken PE, McDevitt A, et al. Manual physical therapy and exercise versus supervised home exercise in the management of patients with inversion ankle sprain: a multicenter randomized clinical trial. J Orthop Sports Phys Ther 2013;43:443-55.

56. Rhon DI, Boyles RB, Cleland JA. One-year outcome of subacromial corticosteroid injection compared with manual physical therapy for the management of the unilateral shoulder impingement syndrome: a pragmatic randomized trial. Ann Intern Med 2014:161:161-9.

57. Sun Y, Sturmer T, Gunther KP, et al. Reliability and validity of clinical outcome measurements of osteoarthritis of the hip and knee--a review of the literature. Clin Rheumatol 1997;16:185-98.

58. Veenhof C, Bijlsma JW, van den Ende $\mathrm{CH}$, et al. Psychometric evaluation of osteoarthritis questionnaires: a systematic review of the literature. Arthritis Rheum 2006;55:480-92.

59. Bellamy N, Buchanan WW, Goldsmith $\mathrm{CH}$, et al. Validation study of WOMAC: a health status instrument for measuring clinically important patient relevant outcomes to antirheumatic drug therapy in patients with osteoarthritis of the hip or knee. J Rheumatol 1988; $15: 1833-40$

60. Juniper EF, Guyatt GH, Willan A, et al. Determining a minimal important change in a disease-specific Quality of Life Questionnaire. $\checkmark$ Clin Epidemiol 1994;47:81-7.
61. Jaeschke R, Singer J, Guyatt GH. Measurement of health status. Ascertaining the minimal clinically important difference. Control Clin Trials 1989;10:407-15.

62. Fritz JM, Irrgang JJ. A comparison of a modified Oswestry Low Back Pain Disability Questionnaire and the Quebec Back Pain Disability Scale. Phys Ther 2001;81:776-88.

63. Dobson F, Hinman RS, Hall M, et al. Measurement properties of performance-based measures to assess physical function in hip and knee osteoarthritis: a systematic review. Osteoarthr Cartil 2012;20:1548-62.

64. Stratford PW, Kennedy DM, Woodhouse LJ. Performance measures provide assessments of pain and function in people with advanced osteoarthritis of the hip or knee. Phys Ther 2006;86:1489-96.

65. Dobson F, Hinman RS, Roos EM, et al. OARSI recommended performance-based tests to assess physical function in people diagnosed with hip or knee osteoarthritis. Osteoarthr Cartil 2013;21:1042-52.

66. Podsiadlo D, Richardson S. The timed "Up \& Go": a test of basic functional mobility for frail elderly persons. J Am Geriatr Soc 1991;39:142-8.

67. Shumway-Cook A, Brauer S, Woollacott M. Predicting the probability for falls in community-dwelling older adults using the Timed Up \& Go Test. Phys Ther 2000;80:896-903.

68. Steffen TM, Hacker TA, Mollinger L. Age- and gender-related test performance in community-dwelling elderly people: Six-Minute Walk Test, Berg Balance Scale, Timed Up \& Go Test, and gait speeds. Phys Ther 2002;82:128-37.

69. Angst F, Aeschlimann A, Stucki G. Smallest detectable and minimal clinically important differences of rehabilitation intervention with their implications for required sample sizes using WOMAC and SF-36 quality of life measurement instruments in patients with osteoarthritis of the lower extremities. Arthritis Rheum 2001;45:384-91.

70. Faul F LA-G, Buchner A. G* Power.

71. Beeton KL, Maffey J, Pool L, et al. Educational standards in orthopaedic manipulative therapy, 2013. 


\section{Correction}

Deyle GD, Gill NW, Rhon DI, et al. A multicentre randomised, 1-year comparative effectiveness, parallel-group trial protocol of a physical therapy approach compared to corticosteroid injections. BMJ Open 2016;6:e010528. The title of this paper is incorrect and should be: A multicenter randomised, 1-year comparative effectiveness, parallel-group trial protocol of a physical therapy approach compared to corticosteroid injection on pain and function related to knee osteoarthritis (PTA Trial).

BMJ Open 2016;6:e010528corr1. doi:10.1136/bmjopen-2015-010528corr1

CrossMark 